\title{
Electronic stucture of methane hydrate studied by Compton scattering
}

\author{
C. Sternemann, ${ }^{1}$ S. Huotari, ${ }^{2}$ M. Hakala,${ }^{3}$ M. Paulus, ${ }^{1}$ M. Volmer, ${ }^{1}$ C. Gutt,,${ }^{1} *$ T. Buslaps,${ }^{2}$ N. Hiraoka, ${ }^{2}$ D. D. Klug, ${ }^{4}$ \\ K. Hämäläinen, ${ }^{3}$ M. Tolan, ${ }^{1}$ and J. S. Tse ${ }^{5}$ \\ ${ }^{1}$ Department of Physics/DELTA, University of Dortmund, D-44221 Dortmund, Germany \\ ${ }^{2}$ European Synchrotron Radiation Facility, Boîte Postale 220, F-38043 Grenoble Cedex, France \\ ${ }^{3}$ Department of Physical Sciences, P.O. Box 64, FI-00014, University of Helsinki, Helsinki, Finland \\ ${ }^{4}$ Stacie Institute of Molecular Science, National Research Council of Canada, Ottawa, Ontario, Canada K1A OR6 \\ ${ }^{5}$ Department of Physics and Engineering Physics, University of Saskatchewan, Saskatoon, Canada S7N 5E2 \\ (Received 13 January 2006; revised manuscript received 6 March 2006; published 3 May 2006)
}

\begin{abstract}
High-resolution Compton scattering spectra of methane, methane hydrate, and ice were measured using incident photon energy of $56.4 \mathrm{keV}$ at beamline ID15B of the European Synchrotron Radiation Facility. The experimental Compton profiles are compared to calculations employing density-functional theory using model atomic clusters. The hydrate has a cagelike structure built up from water molecules and the related Compton profile is observed to change apparently when compared to hexagonal ice. Furthermore, the influence of the guest-host interactions between the methane molecules and the water molecules of the cages on the Compton profile is discussed.
\end{abstract}

DOI: 10.1103/PhysRevB.73.195104

PACS number(s): 78.70.Ck, 32.80.Cy, 61.25.Em

\section{INTRODUCTION}

Clathrate hydrates are nonstoichiometric inclusion compounds with water cagelike structures where gaseous atoms or molecules (guests) can be enclathrated, normally at high pressure and low temperature. There has been considerable fundamental and practical interest in the properties of these clathrate hydrates. A particularly important guest molecule is methane. Large deposits of natural methane hydrate have been found on the ocean floor and in the earth's permafrost regions. ${ }^{1}$ The large accumulation of methane hydrate can be exploited as a potential energy resource and may have significant impact on the global methane budget. At pressures of 50-100 bar, methane and water form the cubic type-I structure, ${ }^{2}$ which consists of eight water cages where each cage contains one methane molecule $\left[\left(\mathrm{CH}_{4}\right)_{8}\left(\mathrm{H}_{2} \mathrm{O}\right)_{46}\right]$. The space group of this structure is $\mathrm{Pm} 3 n$ with a lattice constant of $11.83 \AA$.

The detailed mechanism for formation of gas hydrates is still unknown. It may be considered as a special example of the hydrophobic effect, which emphasizes the importance of guest-host interactions in these hydrate systems. Empty hydrates without guests do not exist because the hydrates are stabilized by the repulsive interaction between the cage and the included guest. ${ }^{3}$ Therefore, gas hydrates are good model systems to study hydrophobic hydration ${ }^{4}$ and water-guest potentials. ${ }^{5}$ Since hydrates consist mostly of hydrogenbonded water molecules, most of their physical properties are similar to those of hexagonal ice. One notable exception is the thermal conductivity which is glasslike despite the crystalline structure of the gas hydrates. ${ }^{6}$ Inelastic x-ray scattering experiments have shown that this behavior could be related to a symmetry-avoided crossing of the acoustic lattice phonons with the localized modes of the guest molecules ${ }^{7}$ in favor of a resonant-scattering mechanism for guest-host phonon interactions. However, the questions of how the guest molecules affect the electronic structure and bonding properties of the water network and how the electronic ground state of the water molecules in the cagelike structure compares to that of hexagonal ice have not been investigated so far.

Compton scattering is a powerful tool to study a wide range of questions related to electronic structure, Fermi surfaces, and bonding properties, because it yields information on the ground-state electron momentum density of the system and is highly sensitive to the change of ground-state wave functions. A comprehensive overview on x-ray Compton scattering is given in Refs. 8 and 9, for example. During the last decade the Compton scattering technique has been applied to increasingly complex materials, such as quasicrystals, perovskites, and fullerenes. ${ }^{10-12}$ Also temperature ${ }^{13,14}$ and pressure effects ${ }^{12,15,16}$ on Compton profiles have been studied. Recently, Itou et al. ${ }^{17}$ reported on the Compton scattering experiments on covalently bonded polycrystalline $\mathrm{Ba}_{8} \mathrm{Si}_{46}$ clathrate, a compound which is isostructural to the hydrates. They found evidence of a charge transfer from the $\mathrm{Ba}$ guest atoms to the $\mathrm{Si}$ host lattice. $\mathrm{Ba} 6 s$ electrons are transferred to $\mathrm{Ba} 5 d$ orbitals which are strongly hybridized with the Si $3 p$ orbitals. Over the last few years highresolution Compton scattering has also been used to study weakly bonded systems such as hexagonal ice, ${ }^{18-20}$ liquid water, ${ }^{20-23}$ and salt solutions. ${ }^{24}$ In these studies important information concerning the effects of bond lengths, bond angles, and hybridization on the electronic properties is obtained.

In this paper we present a combined experimental and theoretical study of the Compton profile of methane hydrate. The outline of the paper is as follows. In Sec. II the theoretical approach for the calculation of the Compton profiles is described. The experiment and the data analysis are discussed in Sec. III followed by the presentation of the results along with a detailed discussion in Sec. IV. Finally, conclusions and a short outlook are given. 


\section{CALCULATION}

The Compton profile $J\left(p_{z}\right)$ is proportional to the doubledifferential scattering cross section in an inelastic x-ray scattering experiment. ${ }^{8,9}$ Within the impulse approximation ${ }^{25}$ it is directly related to the ground-state electron momentum density $n(\mathbf{p})$,

$$
J\left(p_{z}\right)=\iint n(\mathbf{p}) d p_{x} d p_{y},
$$

where the direction of the momentum transfer $\mathbf{q}$ in the experiment is chosen parallel to the $z$ component of the momentum of the scattered electron in its ground state. The electron momentum density is described by the absolute square of the electron wave function in momentum space due to

$$
n(\mathbf{p})=\sum_{\nu}\left|\int d \mathbf{r} \Psi_{\nu}(\mathbf{r}) e^{(i / \hbar) \mathbf{p} \cdot \mathbf{r}}\right|^{2}
$$

with the sum over the occupied single-particle states of electrons.

In this work the electron momentum density is calculated using model atomic clusters within the Kohn-Sham (KS) density-functional theory (DFT) utilizing Gaussian-type orbitals for the electron states. ${ }^{21} \mathrm{~A}$ triple- $\zeta$ valence plus polarization type basis set is used for oxygen and carbon, while for hydrogen a primitive $\operatorname{set}^{26}$ augmented by one $p$ function in a $[3 s, 1 p]$ contraction is employed. A gradient-corrected exchange-correlation functional ${ }^{27,28}$ is used in the calculations. Since the systems under study are isotropic, the calculated Compton profiles are averaged over the different orientations of the scattering vector relative to the cluster geometry. The calculated Compton profiles are also convoluted with the experimental resolution function to enable a direct comparison with the experimental data.

The free methane molecule is assumed to have the $T_{d}$ point-group symmetry and the C-H bond length of $1.091 \AA$ (Ref. 2). To simulate hexagonal ice $\mathrm{I} h$, the methane hydrate, and the empty hydrate cage, a cluster of $N=46$ water molecules is employed. The methane molecule inside the hydrate is assumed to preserve its $T_{d}$ symmetry with the $\mathrm{C}-\mathrm{H}$ bond length of $1.091 \AA$. The clathrate structure has a mean covalent $\mathrm{O}-\mathrm{H}$ bond distance of $1.002 \AA$ and a bond angle of $104.5^{\circ}$. The mean hydrogen bond distance is $1.746 \AA$. The ice $\mathrm{I} h$ structure is assumed to have the same internal $\mathrm{H}_{2} \mathrm{O}$ molecule structure and lattice constants $a=4.5006 \AA$ and $c=7.3341 \AA$. The use of a finite-size-cluster model may introduce some error in the description of the hydrogen-bond network due to the fictitious-surface effects (i.e., molecules on the surface of the cluster do not have the proper nearestneighbor configuration) that may transfer to uncertainties in the calculated absolute Compton profiles. For a water dimer, there is a further uncertainty in the region of $\sim 0.2-2 \%$ of $J(0)$ when the KS DFT calculations are compared to HartreeFock (HF) and Møller-Plesset second-order (MP2) perturbation theory calculations. ${ }^{21,29}$ The KS-DFT method is found to give a larger value of $J(0)$ compared to the HF and MP2 methods. ${ }^{29}$ However, when taking Compton profile differ- ences, these errors cancel out to a large extent. ${ }^{21,29}$ Similarly, errors due to the use of finite-size clusters can be expected to be minimized when Compton profile differences are compared.

\section{EXPERIMENT}

Compton profile measurements of methane hydrate and the references, hexagonal ice and methane, were accomplished at the beamline ID15B of the European Synchrotron Radiation Facility (ESRF) utilizing the high-resolution Compton scattering setup. ${ }^{30}$ The measurements were performed at an incident energy of $56.4 \mathrm{keV}$, a scattering angle of $173^{\circ}$, and with a momentum space resolution at the maximum of the Compton profile of $\Delta p_{z}=0.26$ a.u. ( 0.36 a.u. in the case of methane).

The Compton profile of gaseous methane $(99.9 \%$ pure methane from Air Liquide) was measured at room temperature and at a gas pressure of 50 bar utilizing a special pressure cell. The same setup has been successfully used also before in previous measurements of Compton profiles of several gases. ${ }^{31}$ The modest pressure was used merely to increase the count rate from the low density gases and is not expected to affect the electronic structure of the methane. The pressure cell had an inner volume of $93 \mathrm{~cm}^{3}$. The incident and scattered radiation passed through a $1 \mathrm{~mm}$ thick $\mathrm{Al}$ window. The direct beam was stopped inside the chamber by a specially designed $\mathrm{Pb}$ beam stopper in order to reduce the background and multiple scattering. The experimental spectra were corrected for the self-absorption in methane and for the absorption due to the $\mathrm{Al}$ window. The combined use of a focusing crystal spectrometer and careful slitting in front of both the sample cell and the detector allowed the recording of pure scattering from the gas with a negligible contribution from the cell window. This was verified by a separate measurement using an evacuated cell.

The methane hydrate and ice samples were cooled to $80 \mathrm{~K}$ using a different closed-cycle cryostat setup since the hydrate structure is stable only below $150 \mathrm{~K}$. The methane hydrate samples were synthesized and characterized at the National Research Council of Canada as described elsewhere. ${ }^{32}$ To avoid condensation and decomposition the samples were stored in liquid nitrogen prior to the experiment. The methane hydrate samples for the Compton scattering experiment were prepared by grinding the hydrate into a fine powder. The powder was transferred onto a liquidnitrogen-cooled copper block and then attached to a copper sample holder. This sample holder was mounted on the precooled cryostat. The entire sample preparation procedure was conducted under boiling nitrogen. The integrity of the in situ sample was checked before and after the experiment with $\mathrm{x}$-ray diffraction using a MAR345 image plate detector. The diffraction patterns showed no degeneration of either sample during the measurements. Furthermore, no preferred crystallographic orientation of the hydrate and ice I $h$ was observed. The diffraction data were analyzed using the program packages FIT2D (Ref. 33) and FULLPROF. ${ }^{34}$ From the Rietveld refinement, it was found that all cages were occupied by one methane molecule resulting in an occupation number of 
TABLE I. The thickness of entrance windows, as well as their level of absorption at $p_{z}=0$ (combined for entrance and exit radiation), the effective thicknesses of the samples, sample densities, and level of absorption within the sample for the incident beam, the relative effect of the absorption correction (combined effect of sample self-absorption and window absorption) between $p_{z}=5$ and -5 a.u. of momentum, the total amount of multiple scattering as estimated by a ray-tracing simulation, and finally the momentum space resolution $\Delta p_{z}$.

\begin{tabular}{lcc}
\hline \hline & $\mathrm{CH}_{4}(50 \mathrm{bar})$ & Ice/Hydrate \\
\hline Window $(\mathrm{mm})$ & $1.0(\mathrm{Al})$ & 0.025 (Kapton) \\
Window absorption $(\%)$ & 18.0 & 0.33 \\
Sample thickness $(\mathrm{mm})$ & 30.0 & 3.0 \\
Density $\left(\mathrm{g} / \mathrm{cm}^{3}\right)$ & 0.036 & $0.912 / 0.916$ \\
Sample absorption $(\%)$ & 2.3 & 5.8 \\
Absorption correction $(\%)$ & 2.9 & 0.29 \\
Multiple scattering $(\%)$ & 1.9 & 3.6 \\
$\Delta p_{z}$ (a.u.) & 0.36 & 0.26 \\
\hline \hline
\end{tabular}

$x=8$. The methane hydrate sample contained an ice impurity which was quantified by the Rietveld analysis to be $9.45 \%$. For the Compton scattering measurements several single spectra were collected for each sample and the results were summed up. The background signal was measured separately and subtracted from the sample spectra. At the Compton peak a maximum of about $5 \times 10^{5}$ counts was obtained resulting in a statistical accuracy of $0.15 \%$. After previously described absorption corrections the experimental data were also corrected for the vertical acceptance of the spectrometer and the reflectivity of the analyzer crystal. Furthermore, a scale correction was applied and the relativistic scattering cross-section correction was performed. Finally, the spectra were converted to momentum scale and properly normalized to the number of electrons (ten electrons for methane and for ice). In order to obtain the Compton profile of pure methane hydrate, $J_{\mathrm{mh}}^{\text {expt }}\left(p_{z}\right)$, the contribution of the small portion of ice was subtracted from the measured total Compton profile, $J_{\text {mhtice }}^{\text {expt }}\left(p_{z}\right)$, using the measured Compton profile of ice, $J_{\text {ice }}^{\text {expt }}\left(p_{z}\right)$, via $J_{\mathrm{mh}}^{\text {expt }}\left(p_{z}\right)=\left[J_{\mathrm{mh}+\text { ice }}^{\text {expt }}\left(p_{z}\right)-b \times J_{\text {ice }}^{\text {expt }}\left(p_{z}\right)\right] /(b-1)$ with $b=9.45 \%$ obtained from the diffraction analysis as described above. The methane hydrate Compton profile was normalized to 11.74 electrons considering ice as a reference and assuming that each ice cage is occupied by one methane molecule (ten electrons for $\mathrm{H}_{2} \mathrm{O}$ and $8 / 46 \times 10$ electrons for $\mathrm{CH}_{4}$ ). Within Table I some characteristics of the different experimental setups are summarized. A general overview on the application of correction factors in the analysis of Compton scattering can be found in Ref. 9. The experimental Compton profiles of ice, methane, and methane hydrate will be discussed in detail in the following section.

\section{RESULTS AND DISCUSSION}

The measured Compton profiles of methane and ice are compared to the calculated Compton profiles in Fig. 1. The figure shows also the computed weighted sum of the meth-

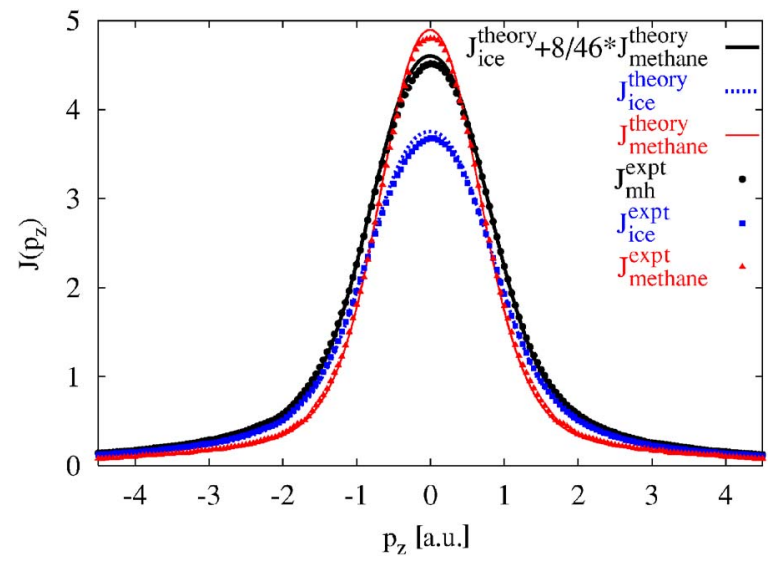

FIG. 1. (Color online) Experimentally obtained Compton profiles of methane hydrate (dots), ice (squares), and methane (triangles) compared to the corresponding calculations (thick solid, dashed, and thin solid lines, respectively).

ane and ice Compton profiles $J_{\text {ice }}^{\text {theory }}\left(p_{z}\right)+8 / 46 \times J_{\text {methane }}^{\text {theory }}\left(p_{z}\right)$ together with the measured methane hydrate Compton profile $J_{\mathrm{mh}}^{\text {expt }}\left(p_{z}\right)$. The computed Compton profile is higher than the experimental one in the vicinity of the Compton peak $\left(p_{z}=0\right)$ but is lower than the experiment for larger values of $p_{z}$. The difference at the Compton peak is about $2 \%$ and could be related to a deficiency of the KS DFT method in predicting quantitatively the peak height of the absolute Compton profiles as discussed in Sec. II. ${ }^{21,29}$

In order to analyze the Compton profile of methane hydrate the theoretical and the experimental reference spectra of ice and methane will be used to illustrate the effect of both the guest-host interactions and the cagelike arrangement of water molecules. Since the Compton profiles of methane hydrate and hexagonal ice were measured at the same experimental conditions, their spectra can be compared directly. However, the Compton profile of pure methane was measured in a different sample environment (using a gas cell) and resulted in a different momentum space resolution. The first step to model the Compton profile of the methane hydrate is to calculate the sum of the experimental Compton profile of ice, $J_{\text {ice }}^{\text {expt }}\left(p_{z}\right)$, and the calculated Compton profile of methane, $J_{\text {methane }}^{\text {theory }}\left(p_{z}\right)$, with $J_{\text {mh }}^{\text {model }}\left(p_{z}\right)$ $=J_{\text {ice }}^{\text {expt }}\left(p_{z}\right)+8 / 46 \times J_{\text {methane }}^{\text {thery }}\left(p_{z}\right)$. This model assumes that the Compton profile of the hydrate can be mimicked as a simple superposition of two independent and noninteracting constituents and that the Compton profile of ice is the same as the one of the empty hydrate. The latter assumption may be justified in a first approximation since both systems consist of networks of hydrogen-bonded water molecules. In a more detailed analysis the different arrangement of the water molecules must be taken into account. The experimental Compton profile of methane was not used in this analysis. Because the measurements of methane were performed under different experimental conditions, a direct modeling of the experimental methane hydrate Compton profile by a superposition of the exprimental ice and methane Compton profiles is not appropriate. It is important to note that the occupancy of methane in the hydrate can be extracted from the experimental Compton profile $J_{\mathrm{mh}}^{\mathrm{expt}}\left(p_{z}\right)$ of methane 

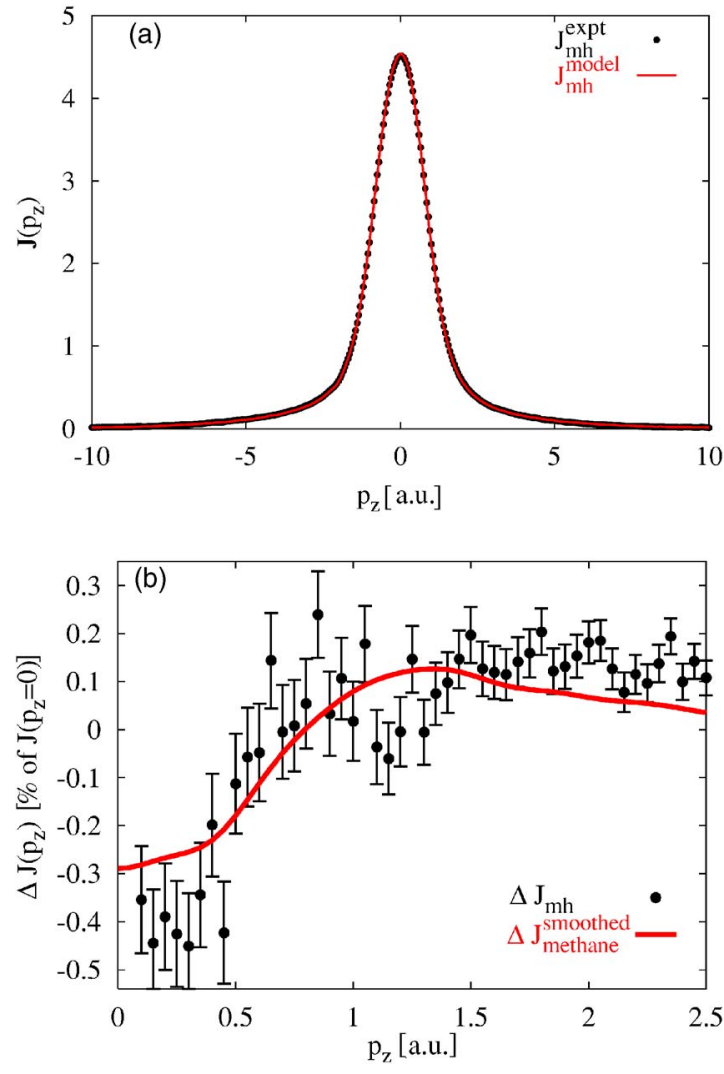

FIG. 2. (Color online) (a) Experimental Compton profile of methane hydrate (dots) compared to the model as discussed in the text (solid line). (b) Symmetrized difference between experiment and model (dots with error bars) compared to the smoothed difference between experiment and theory for methane (solid line; see Fig. 1 for original data).

hydrate by assuming that the contributions from ice (experiment) and methane (theory) are additive, $J_{\mathrm{mh}}^{\text {expt }}\left(p_{z}\right)$ $=J_{\text {ice }}^{\text {expt }}\left(p_{z}\right)+x_{\text {fit }} / 46 \times J_{\text {methane }}^{\text {theory }}\left(p_{z}\right)$. The fit yields a value of $x_{\mathrm{fit}}=7.94$ in excellent agreement with $x=8$ obtained from the Rietveld analysis of the diffraction data.

The Compton profile of methane hydrate, $J_{\mathrm{mh}}^{\mathrm{expt}}\left(p_{z}\right)$, is compared with the model, $J_{\mathrm{mh}}^{\text {model }}\left(p_{z}\right)$, in Fig. 2(a). A good overall agreement between the experiment and model is found. Careful examination, however, reveals some subtle differences. The model is found to predict slightly higher values for the Compton profile for $p_{z}<0.7$ a.u. This trend is reversed for larger values of $p_{z}$. These discrepancies are beyond the statistical accuracy of the measurement and they become more apparent if the corresponding symmetrized Compton profile difference normalized to the maximum value of the experimental Compton profile of the methane hydrate $\Delta J_{\mathrm{mh}}\left(p_{z}\right)=\left[J_{\mathrm{mh}}^{\mathrm{expt}}\left(p_{z}\right)-J_{\mathrm{mh}}^{\mathrm{model}}\left(p_{z}\right)\right] / J_{\mathrm{mh}}^{\text {expt }}(0)$ is plotted as shown in Fig. 2(b). The Compton profile difference $\Delta J_{\mathrm{mh}}\left(p_{z}\right)$ could be related to the following effects: (i) the discrepancy between the experimental and computed Compton profiles of methane (owing to the different experimental setups, the theoretical Compton profile of methane has to be used in the model instead of the experimental one); (ii) the different local arrangement of water molecules in the hydrate cagelike structure and the ice I $h$ structure; (iii) the influence of the

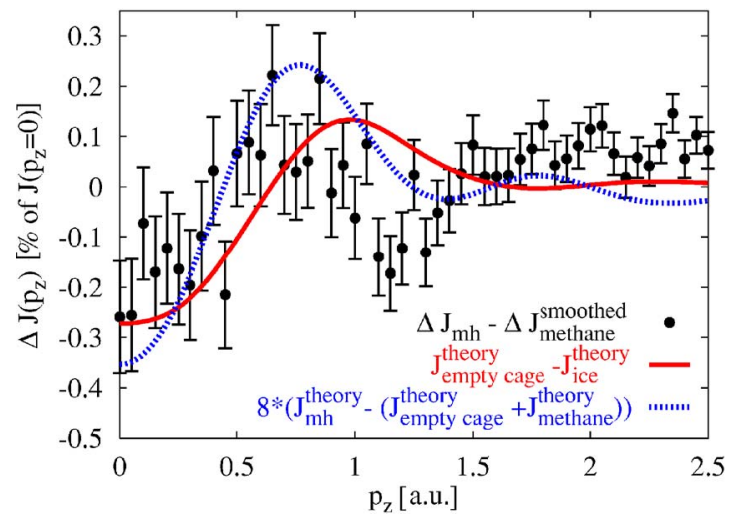

FIG. 3. (Color online) Difference between $\Delta J_{\mathrm{mh}}\left(p_{z}\right)$ and $\Delta J_{\text {methane }}^{\text {smoothed }}\left(p_{z}\right)$ compared to the difference between computed Compton profiles of the empty cage hydrate and ice (solid line) which accounts for the different local arrangement of water molecules in the hydrate structure compared to ice [discussed under (ii) within the text]. Moreover, the difference between methane hydrate and the sum of the empty cage and methane weighted by a factor 8 is shown (dashed line) which describes the influence of the guest-host interaction [discussed under (iii) within the text].

interaction between the $\mathrm{H}_{2} \mathrm{O}$ host lattice and the methane guest molecules.

It is demonstrated below that (i) is largely responsible for the difference in the symmetrized Compton profiles. The difference between the experimental and the theoretical Compton profile of methane $\Delta J_{\text {methane }}\left(p_{z}\right)=\left[J_{\text {methane }}^{\text {expt }}\left(p_{z}\right)\right.$ $\left.-J_{\text {methane }}^{\text {theory }}\left(p_{z}\right)\right] / J_{\mathrm{mh}}^{\text {expt }}(0)$, weighted by a factor of $8 / 46$ which accounts for the proper normalization of the Compton profiles with methane hydrate, is depicted in Fig. 2(b). The solid line represents a smoothed curve of $\Delta J_{\text {methane }}\left(p_{z}\right)$ and shows good overall agreement with $\Delta J_{\mathrm{mh}}\left(p_{z}\right)$. Therefore, the Compton profile of methane hydrate can be described reasonably well by a straightforward superposition of the Compton profiles of methane and ice. This observation indicates that the effects related to points (ii) and (iii) are relatively small.

Even though the contributions from (ii) and (iii) to the Compton profile are expected to be small, nevertheless, their effects can be estimated by calculating the difference of $\Delta J_{\text {mh }}\left(p_{z}\right)$ and $\Delta J_{\text {methane }}^{\text {smoothed }}\left(p_{z}\right)$. The results are shown in Fig. 3 and indicate a maximum negative amplitude of about $0.2 \%$ at the Compton peak.

The change of the Compton profiles due to the different arrangement of the water molecules in a structure-I hydrate and hexagonal ice can be examined by calculation of the Compton profile difference between theoretical empty cage $J_{\text {empty cage }}^{\text {theory }}\left(p_{z}\right)$ and hexagonal ice $J_{\text {ice }}^{\text {theory }}\left(p_{z}\right)$ computational results. This difference accounts directly for (ii) and is shown in Fig. 3. The principal feature of $J_{\text {empty cage }}^{\text {theory }}\left(p_{z}\right)-J_{\text {ice }}^{\text {theory }}\left(p_{z}\right)$ is in qualitative agreement with the experiment. The curve shows a similar pattern, negative values for small $p_{z}$ and becoming positive for $p_{z}>0.65$ a.u. Moreover, the absolute magnitude is also in good agreement with the experiment. However, a significant difference is that the experimental difference changes its sign at $p_{z} \approx 0.5$ a.u., whereas the calculated curve changes its sign at $p_{z}=0.65$ a.u. The guest-host interactions (iii) can be also estimated by calculation of the 
difference between theoretical methane hydrate Compton profile $J_{\mathrm{mh}}^{\text {theory }}\left(p_{z}\right)$ with the sum of the theoretical empty cage and methane Compton profiles $J_{\text {empty cage }}^{\text {theory }}\left(p_{z}\right)+J_{\text {methane }}^{\text {theory }}\left(p_{z}\right)$ weighted by a factor 8 for a proper normalization of the guest-host interactions per unit cell. As shown in Fig. 3, the calculated difference is in better agreement with the experimental results. In particular, a change in sign of this difference is found at $p_{z}=0.45$ a.u., which is close to the value obtained by the experiment. This indicates that the total bonding properties of methane hydrate, i.e., (ii) the bonding properties of the empty cage versus ice (represented by the solid curve in Fig. 3) and (iii) the bonding properties of methane inside the cage (dashed curve in Fig. 3), may be importantly influenced by the guest-host interactions (iii). This net interaction is repulsive and due to the exchange repulsion between the filled electron shells of methane and its water molecule surroundings, leading to a characteristic oscillatory feature in the Compton profile. ${ }^{21,35}$

In summary, the main features in the experimental Compton profile difference shown in Fig. 3 can be explained by both the difference in the electronic properties of water in the hydrate cage structure compared with ice $\mathrm{I} h$ and by guesthost interactions. Despite the fact that the statistical accuracy is of the same order of magnitude as the predicted effects, it is difficult to draw a final conclusion. Nevertheless, the results indicate that both the properties (ii) and (iii) affect the Compton profile with roughly the same order of magnitude. The dip that appears in the experimental difference close to $p_{z}=1.2$ a.u. is not reproduced by theory in both cases and its origin is not understood so far. Subtle structural changes, such as changes in the internal $\mathrm{O}-\mathrm{H}$ bond lengths of the water molecules or in the $\mathrm{C}-\mathrm{H}$ lengths of methane, may be necessary to take into account to explain the residual differences. Furthermore, in order to study the weaker effects (ii) and (iii) in more detail, a continuation of such Compton scattering measurements with higher statistical accuracy and of different types of hydrates, e.g., with noble gas guest atoms, is needed. Overall it has been demonstrated that the methane hydrate Compton profile can be satisfactorily modeled by a simple superposition of the sum of the Compton profiles of its constituents with minor contributions to guest-host interactions and the distortion of water forming the clathrate lattice.

\section{CONCLUSION}

Compton profiles of methane hydrate, ice, and methane have been measured and compared with computed results utilizing density-functional theory. It has been shown that the Compton profile of methane hydrate could be modeled by a simple sum of the Compton profiles of its constituents, methane and ice. The small discrepancies related to the bonding properties which could not be explained by this simple model are traced back to the different local arrangement of water molecules in hexagonal ice compared to the cagelike structure of the hydrate, as well as to the guest-host interactions. Weak hints as to how these interactions manifest themselves in the Compton profile differences are illustrated. The repulsive interaction between the methane guest molecules and the host lattice led to an oscillatory feature in the Compton profile which, in principle, can be detected experimentally.

\section{ACKNOWLEDGMENTS}

The authors acknowledge the ESRF for providing synchrotron radiation facilities. We would like to thank J. Baumert for expert advice during the preparation of the samples for the cryostat environment. M.P. is grateful for the financial support by the Graduiertenkolleg GK298 "Struktur und Dynamik Beziehungen in mikrostrukturierten Systemen" at the University of Dortmund. C.S. would like to thank W. Schülke for stimulating discussions. M.H and K.H. are supported by the Academy of Finland (Contracts No. 201291, No. 205967, and No. 110571) and the Research Funds of the University of Helsinki. M.H. is supported by the Academy of Finland through its Centers of Excellence program.
*Present address: HASYLAB, DESY, Notkestrasse 85, D-22607, Hamburg, Germany.

${ }^{1}$ E. D. Sloan, Clathrate Hydrates of Natural Gases (Marcel Dekker, New York, 1990).

${ }^{2}$ C. Gutt, B. Asmussen, W. Press, M. R. Johnson, Y. P. Handa, and J. S. Tse, J. Chem. Phys. 113, 4713 (2000).

${ }^{3}$ P. M. Rodger, J. Phys. Chem. 94, 6080 (1990).

${ }^{4}$ D. T. Bowron, A. Filipponi, M. A. Roberts, and J. L. Finney, Phys. Rev. Lett. 81, 4164 (1998).

${ }^{5}$ J. S. Loveday, R. J. Nelmes, M. Guthrie, D. D. Klug, and J. S. Tse, Phys. Rev. Lett. 87, 215501 (2001).

${ }^{6}$ R. G. Ross, P. Andersson, and G. Bäckström, Nature (London) 290, 322 (1981).

${ }^{7}$ J. Baumert, C. Gutt, V. P. Shpakov, J. S. Tse, M. Krisch, M. Müller, H. Requardt, D. D. Klug, S. Janssen, and W. Press, Phys. Rev. B 68, 174301 (2003).
${ }^{8}$ M. J. Cooper, Rep. Prog. Phys. 48, 415 (1985).

${ }^{9}$ M. J. Cooper, P. E. Mijnarends, N. Shiotani, N. Sakai, and A. Bansil, X-ray Compton Scattering (Oxford University Press, New York, 2004).

${ }^{10}$ J. T. Okada, Y. Watanabe, S. Nanao, R. Tamura, S. Takeuchi, Y. Yokoyama, N. Hiraoka, M. Itou, and Y. Sakurai, Phys. Rev. B 68, 132204 (2003).

${ }^{11}$ N. Hiraoka, T. Buslaps, V. Honkimäki, H. Minami, and H. Uwe, Phys. Rev. B 71, 205106 (2005), and references therein.

${ }^{12}$ A. A. Sabouri-Dodaran, Ch. Bellin, M. Marangolo, G. Loupias, S. Rabii, F. Rachdi, Th. Buslaps, and M. Mezouar, Phys. Rev. B 72, 085412 (2005), and references therein.

${ }^{13}$ C. Sternemann, T. Buslaps, A. Shukla, P. Suortti, G. Döring, and W. Schülke, Phys. Rev. B 63, 094301 (2001).

${ }^{14}$ K. Nygård, S. Huotari, K. Hämäläinen, S. Manninen, T. Buslaps, N. H. Babu, M. Kambara, and D. A. Cardwell, Phys. Rev. B 69, 
020501(R) (2004).

${ }^{15}$ K. Hämäläinen, S. Huotari, J. Laukkanen, A. Soininen, S. Manninen, C.-C. Kao, T. Buslaps, and M. Mezouar, Phys. Rev. B 62, R735 (2000).

${ }^{16}$ J. S. Tse, D. D. Klug, D. Jiang, C. Sternemann, M. Volmer, S. Huotari, N. Hiraoka, V. Honkimäki, and K. Hämäläinen, Appl. Phys. Lett. 87, 191905 (2005).

${ }^{17}$ M. Itou, Y. Sakurai, M. Usuda, C. Cros, H. Fukuoka, and S. Yamanaka, Phys. Rev. B 71, 125125 (2005).

${ }^{18}$ E. D. Isaacs, A. Shukla, P. M. Platzman, D. R. Hamann, B. Barbiellini, and C. A. Tulk, Phys. Rev. Lett. 82, 600 (1999).

${ }^{19}$ S. Ragot, J.-M. Gillet, and P. J. Becker, Phys. Rev. B 65, 235115 (2002).

${ }^{20}$ K. Nygård, M. Hakala, S. Manninen, A. Andrejczuk, M. Itou, Y. Sakurai, A. Nilsson, L. G. M. Pettersson, and K. Hämäläinen (unpublished).

${ }^{21}$ M. Hakala, S. Huotari, K. Hämäläinen, S. Manninen, Ph. Wernet, A. Nilsson, and L. G. M. Pettersson, Phys. Rev. B 70, 125413 (2004).

${ }^{22}$ M. Hakala, K. Nygård, S. Manninen, S. Huotari, T. Buslaps, A. Nilsson, L. G. M. Pettersson, and K. Hämäläinen (unpublished).

${ }^{23}$ M. Hakala, K. Nygård, S. Manninen, L. G. M. Pettersson, and K.
Hämäläinen, Phys. Rev. B 73, 035432 (2006).

${ }^{24}$ K. Nygård, M. Hakala, S. Manninen, K. Hämäläinen, M. Itou, A. Andrejczuk, and Y. Sakurai, Phys. Rev. B 73, 024208 (2006).

${ }^{25}$ P. Eisenberger and P. M. Platzman, Phys. Rev. A 2, 415 (1970).

${ }^{26}$ S. Huzinaga, J. Chem. Phys. 42, 1293 (1965).

${ }^{27}$ J. P. Perdew, K. Burke, and M. Ernzerhof, Phys. Rev. Lett. 77, 3865 (1999).

${ }^{28}$ B. Hammer, L. B. Hansen, and J. K. Norskov, Phys. Rev. B 59, 7413 (1999).

${ }^{29}$ T. Pylkkänen (private communication).

${ }^{30}$ P. Suortti, T. Buslaps, P. Fajardo, V. Honkimäki, M. Kretzschmer, U. Lienert, J. E. McCarthy, M. Renier, A. Shukla, Th. Tschentscher, and T. Meinander, J. Synchrotron Radiat. 6, 69 (1999).

${ }^{31} \mathrm{~S}$. Huotari (private communication).

${ }^{32}$ Y. P. Handa and J. G. Cook, J. Phys. Chem. 81, 6327 (1987).

${ }^{33}$ A. P. Hammersley, Synchrotron Radiat. News 2, 24 (1989).

${ }^{34}$ J. Rodriguez-Carvajal, Proceedings of the Satellite Meeting on Powder Diffraction of the XV Congress of the IUCr, Toulouse, France, 1990 (private communication), p. 127.

${ }^{35}$ T. K. Ghanty, V. N. Staroverov, P. R. Koren, and E. R. Davidson, J. Am. Chem. Soc. 122, 1210 (2004). 